\title{
An Improved Brain Storage Model
}

\author{
Kang Feng \\ School of Computer, Huainan Normal University \\ Huainan 232038, China \\ Email:15855432472@163.com
}

\begin{abstract}
In order to correct the shortcomings of the former brain storage model, an improved brain storage model was proposed, it consisted of a memory bank and a series of sub-storages. These substorages were concluding sub-storage, effective search sub-storage, storing sub-storage, recalls sub-storage and forgetting sub-storage. The input formation of the improved brain storage model was single fuzzy cognitive unit event. Experimental results demonstrated that the model was able to simulate storage functions of the brain, such as concluding, storing, recalling and forgetting. So the improved brain storage model is optimal.
\end{abstract}

Keywords—single fuzzy cognitive unit event, brain models, perception, memory architecture, cognitive systems.

\section{INTRODUCTION}

The brain storage model is built to simulate the brain's memory, the research of brain storage model will put forward the scientific theories and methods of brain storage, and these theories and methods will be applied in computer science and artificial memory system ${ }^{[1]}$. There are four kinds of brain storage models, they are the brain storage model based on anatomy, the brain storage model based on neuroimaging, the brain storage model based on cognitive science and the brain storage model based on linguistics.

The basic unit which the brain stores cognitive information is neuron in the brain storage model based on anatomy, the main site of cognitive information storage is about 15000000000 neurons. In the brain storage model based on anatomy, the different physical regions of pallium store the different cognitive information, the cognitive information about human intelligence and movement is stored in the frontal lobe of pallium ${ }^{[2]}$ and the cognitive information about human perception ${ }^{[1]}$, perception, and language is stored in the parietal of pallium ${ }^{[1]}$, the cognitive information about human vision is stored in the occidental of pallium ${ }^{[1]}$, and the cognitive information about human auditory or olfactory is stored in the temporal 1 of pallium ${ }^{[3]}$. Therefore, the storage substance of brain storage model based on anatomy is to classify the different nature of cognitive information in different pallium. The brain storage model based on neuroimaging applies modern neuroimaging techniques based on brain function, such as MEG, EEG, ERPS, SPRCT, PET, fMRI and other technologies ${ }^{[4]}$. The cognitive information stored human cognitive activities is stored in the physical location of the brain, thus establishing the corresponding brain storage model; With these techniques, it is found that the

This paper was supported by the Anhui Provincial Natural Science Foundation (1508085MF124). pallium allocates different regions of different types of information or as a temporary storage area for the operation, such as working memory information is stored in the left hemisphere after the parietal cortex(BA 40), when the word working memory is repeated, the left hemisphere Bro ca area(BA 44), the left front motor area(BA 6) and the left supplementary motor area(BA 8) are used as temporary storage area ${ }^{[5]}$, Visual working memory information is stored in the left hemisphere of the parietal and temporal lobe. For long term memory, the pallium also has a corresponding storage area, Such as long term memory's episodic memory is stored in the left frontal lobe, temporal lobe and parietal occidental region, Long term memory's semantic memory is stored in the left superior temporal Cyrus(BA 21) and bilateral superior temporal parietal area(BA 37) in the left frontal and temporal lobes; In this book, the memory of the brain's memory model based on neuroimaging is the memory of the working memory and the memory of the different regions of long term memory in the pallium. The brain storage model based on cognitive science is built by cognitive science experiments. The cognitive science experiments included Poisoner visual information encoding experiment, Clark and Chase sentence picture matching experiment, Gutenberg shortterm memory information adding factor method, character conversion experiment("Open window" experiment), Peterson and Peterson forgetting process experiment ${ }^{[6]}$, Waugh and Norman separate traces of fading and interference experiment, four card card really and falsification, etc. a series of cognitive science experiment. Through these experiments, Baddie found that the information of stimulating human cognition is divided into three kinds of different components of working memory, Chen Shunting memory, and procedural memory, These three kinds of different components, also known as Baddie three components, they are based on working memory information, connotation and extension can be more broad; Therefore, the brain storage model based on cognitive science is a general working memory model based on the Baddie three component model. The brain storage model based on linguistics, which is based on the perspective of the composition and organization of the human language, is a model for mapping the brain; The human brain is born with the recognition and control of the organization of neurons in a syllable. Composed of syllables, words, phrases, phrases, sentences, sentences, sentences, and different ways of training and learning, which are different levels of human language, are stored in the pallium. Therefore, the brain storage model based on linguistics is stored in the brain is a mufti-layer organization rule of language rather than the language itself, these different levels of organizational 
rules (program) in the instinctive syllables (data) on the running result is the language.

These four kinds of brain storage models lack the fusion of different cognitive information in the world. Or only for a kind of cognitive information, such as the language, or to maintain the original format of the cognitive information stored in the storage model directly; And they do not reveal the true cause of the brain's memory to be triggered, mistakenly believed that the perception of information to stimulate the sense organs to trigger the brain storage; In addition, these models do not explicitly store the contents of the data. The four kinds of brain storage models can not accurately simulate the memory mechanism of the brain, they are not suitable for computer science.

\section{AN IMPROVED BRAIN STORAGE MODEL}

The improved brain storage model is based on single fuzzy cognitive unit event, it is shown in Figure 1. It consists of a memory bank and a series of sub-storages. These sub-storages are concluding sub-storage, effective search sub-storage, storing sub-storage, recalls sub-storage and forgetting substorage.

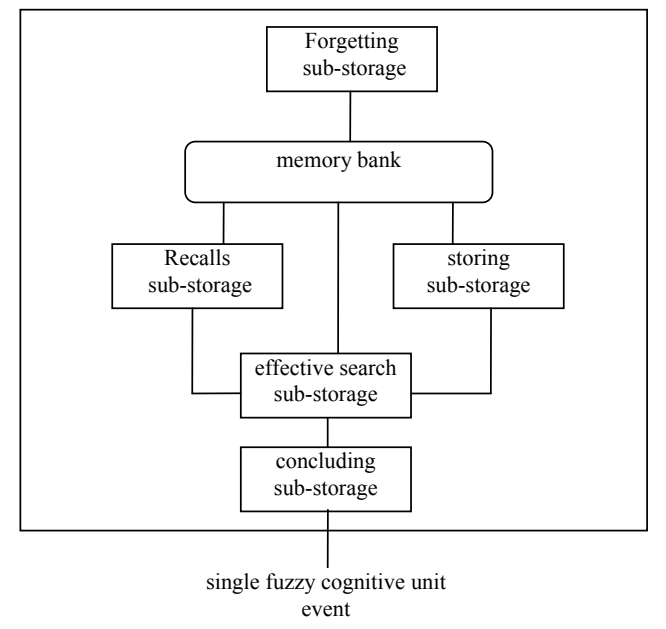

Fig. 1 The improved brain storage model

III. MEMORY BANK

\section{A. Cognition memory}

Memory bank is a form of storage array, the number of rows of tables $l_{y}$ and column number $c_{x}$ is not fixed, according to the memory of the situation at any time to change. Each cell line is used to store and recognize the relevant attributes, contributions, experience factors, ideal cognitive elements, etc. The first line of the first cell stores the an ideal cognitive meta tags $y$, and finally a cell stores the experience factors identification of $m, y$ and $m$ between the rest of the cell store contains all cognition of memory storage will element attribute. If all the assumption contained in the memory storage element will attribute is $n$, the number of columns of memory must be $c_{x}=n+2$. From the beginning of the second row, each row can store a cognition, because everyone knows does not necessarily contain the first line in all the will element attributes, so from the beginning of the second line, some cell stores the contribution degree, some cells without storage with degree. Storing contribution degree, mark $y$ and $m$ and have called for effective cell, and no data storage cells known as invalid cell, the banks will be able to accommodate the effective cell maximum value memory capacity, denoted by $v$, the memory capacity is limited meta properties of the cell. The cell is clearly useless does not occupy the storage capacity of the.

\section{B. Cognition at the bank storage format}

Cognition in memory storage formats starts on the second line, in units, know a stored in each row, the multiple cognition continuous storage. The ideal Fuzzy cognitive element is stored in the most left cell of the row, if it contains a certain element attribute, the row and the row of cells in the column of the element attribute are stored in the contribution of the element attribute. Each awareness bank called in the vertical position recognize clarity Location, denoted by LC, the highest resolution is Top, which is located in the second row of the bank, and then successively lower resolution, namely Top-1, Top-2, ....., the bottom of the lowest resolution cognition, called Bottom, Top value is fixed, always points to the second row of the bank, while the value of Bottom is floating, depending on storage capacity and cognition of the situation of the bank, Top-Bottom +1 is total cognition of storage, so,$l_{y}=$ Top-Bottom +2 . At some point, all of the bank in the total number of valid cells called cognitive capacity $q$ (Quantity), taking into account the total number of cells in each of the new cognition of $c$ stored in the memory banks, used as $d c+2$, so called storage the difference between the amount of capacity and awareness of the body's potential to minus $2 \mathrm{p}$ (Potentiality), obviously, $p=v-q-2$, said that the current bank may also recognize the new storage capacity.

\section{Make full use of the capacity of the bank}

The memory bank know the situation, to change the number of banks and the number of rows and columns, so that the cognitive capacity $q$ as close as possible storage capacity $v$. Make new cognition $c$ storage dimension $d c=j$, if $\mathrm{j} \geq c_{x}$, then $c_{x}$ $=\mathrm{j}, l y=l y+1$; on the contrary, if $\mathrm{j}<c_{x}$, then $c_{x}=c_{x}, l y=l y+1$, enabling storage body ranks variable.

\section{SUB-STORAGE IN THE IMPROVED BRAIN STORAGE MODEL}

\section{A. Effective search sub-storage}

Effective search sub-storage calculate the output subawareness is triggered after induction, the calculation process is beginning to Top of cognition from the bank definition, in accordance with the level of clarity in order to search the storage body is stored there and the associated $c$ corresponding awareness $c_{\mathrm{a}} \propto c$, if the search to recognize the echo of the association and the corresponding definition $c_{\mathrm{a}}$ and $l c$, if the search is complete clarity of cognition still no association Bottom cognition, then sent back a 'false'. Effective search sub-storage can be calculated as follows: 
$C$,

search from Top to next until Bottom,

if $c_{\mathrm{a}} \propto C_{C}$,

then output $c_{\mathrm{a}}$ and $l c$,

exit,

else output 'false',

exit,

\section{B. Storing sub-storage}

Storing sub-storage referred to as sub-storage cognition, its trigger condition is effective search backlog a 'false', indicates that the associated get to know the bank is not and concluding output, then immediately stored in the memory banks of the original cognition of both clarity reduce one unit, namely the bank originally stored in cognition the overall down one line, and then stored in the second row an initial cognition of the bank to clarity as Top.

Storing sub-storage can be calculated as follows:

'false',

all $c$ move down one column,

store $c_{p}$ in Top,

exit,

Storing sub-storage fully simulate the brain cognitive memory function for fresh information.

\section{Recalls sub-storage}

Recalls sub-storage trigger condition is an effective search backlog associated cognition and the corresponding resolution, indicating get to know the bank associated with and summarized in output, the output of the subsequent induction immediately recognize storage in the second row of the bank, so that the clarity of Top, the original is deleted from the memory bank associated with the cognition, cognition associated resolution higher than the original cognition down one unit below remain unchanged.

Recalls sub-storage can be calculated as follows:

$C_{\mathrm{a}} \propto C_{\mathrm{s}}$ and $l c$,

delete $c_{\mathrm{a}} \propto c_{\mathrm{s}}$,

all $c$ of location above lc move down one column,

store $c_{\mathrm{s}}$ in Top,

exit,

Recalls son calculate fully simulate the brain cognitive update information storage function similar has happened in.

\section{Forgetting sub-storage}

Forgetting sub-storage trigger condition during memory, if the initial cognition of dimension greater than the potential that $d c_{\mathrm{p}}>p$, then the lowest resolution will recognize one or more out of the bank. Forgotten is limited by the capacity of the bank caused by forgetting to recognize the loss.

Forgetting sub-storage can be expressed as follows:

$$
\begin{aligned}
& \text { Storing Cognition } \\
& d c_{\mathrm{p}} \text { above } p, \\
& \text { shiftout: } c \text { of Bottom shift out of memory, } \\
& p_{\text {new }}=p+d c+2, \\
& \text { if } d c_{\mathrm{p}} \text { above } p_{\text {new }}, \\
& \text { go to shiftout, } \\
& \text { else exit, }
\end{aligned}
$$

Forgetting sub-storage fully simulate the function of the brain store oblivion without deep cognition of image information, forgetting to remember more fresh cognitive information.

\section{EXPERIMENTS OF THE IMPROVED BRAIN STORAGE MODEL}

To test based on a dollar Fuzzy event simulation model of the brain brain memory storage mechanism, we designed a cognitive science belonging to the five sections of cognitive memory experiment, and the experiment was based on a meta Fuzzy brain store event simulation. The five sections of cognitive memory test is divided into five time periods, each time period in the previous four time periods connected in order to give participants 4 screen different cognitive cases, but 2 screen to recognize various time periods between known case has similarities, contains the same rule. If a case of cognition screen for the first time on screen simultaneously haphazard 6 small red equilateral triangle and four small white squares, then they disappear together, screen then appears 10 small red circle; s a screen cognitive case II period also appear on the screen simultaneously haphazard seven white and five red small square small equilateral triangles, and then they disappeared together, the screen then appears two small red circle; obviously two case 2 screen cognitive similarities period. But the fifth time and the fourth time the lack of a first screen similar cognitive case, but with a screen (referred to as heterogeneous screen) with the previous period in all cases are not similar cognitive cognitive case instead. All experiments cognitive cases have similarities inherent in the law are normal people can be summed up summed up the object experiment is 213 sophomores. We were asked to remember only four law can be summarized, and at the end of the fifth period of rapid write induction remember four law.

The results show: 201 students correctly summarized and remember the four rules, accounting for $94 \%$ of the total sample; wherein 179 students remember the four law contains a heterogeneous screen contains a rule, accounting for the total number of subjects $84 \%$; and in this 179 students summed up the four laws, the 154 does not include the fourth period of the first screen contains the cognitive case law, accounting for $86 \%$ remember the law of heterogeneous screen. Taking into account individual differences and human factors, the results can be summarized as: brain summarized and remember the 
recent case contains four screens cognitive law, but forget the first rule to remember.

Above five sections based cognitive memory test event one yuan Fuzzy brain memory simulation. The five time periods in order to give the subjects of 4 screen different cognitive cases, defined as four one dollar Fuzzy event $e_{i 1}, e_{i 2}, e_{i 3}, e_{i 4}$, ( $i$ for the number of time periods), recognized each time period between known cases have similarities, thus further cognitive case between each period is defined as one dollar Fuzzy mutually similar events. Since the five sections of the storage case limited cognitive subjects just remember the case of 4-screen cognitive meaning, which is equivalent to the subjects after each period of 4 screen different cognitive cognitive cases, the bank's existential is 0 . We put four cases in which a panel of cognitive experiment of all time simulation table one dollar Fuzzy event sequence of the second column, visible, $e_{11} \operatorname{sse}_{24} \operatorname{cse}_{34} \operatorname{cse}_{42} \operatorname{cse}_{54}, e_{12} \operatorname{cse}_{22} \operatorname{cse}_{33}$ $\sim_{e_{44}} \sim_{e_{51}}, e_{13} \sim_{e_{21}} \sim_{e_{32}} \sim_{e_{41}}, e_{14} \sim_{e_{23}} \sim_{e_{31}} \omega_{e_{43}} \omega_{e_{52}}$, fifth period of $e_{51}, e_{52}, e_{54}$ respectively the fourth period of $e_{44}$, $e_{43}, e_{42}$ similar, but the fifth time in paragraph heterogeneous screen corresponding $e_{53}$ with the first event of a dollar Fuzzy fourth period of $e_{41}$ dissimilar.

Computer simulation experiments on all five sections referring to the cognition stored simulation methods. Simulation results were at $t_{4}$ and $t_{5}$ time, the bank stores a four awareness; awareness $t_{5}$ moment, and $e_{41}$ associated forgotten. This result and the five sections of cognitive memory experiment basically. Thus, the brain storage model based on a dollar Fuzzy event to simulate the brain's induction, memory, memories, forgotten and other storage functions.

\section{CONCLUSION}

Brain storage model based on a dollar Fuzzy event will be different cognitive information fusion of Fuzzy cognitive elements form, Fuzzy Cognitive yuan can only be perceived sensory organs, and can not trigger the brain to store, really trigger the brain stores a dollar Fuzzy events occur. Storing data stored in the brain is to recognize the essence of brain memory is calculated by computing a series of memory storage sub-constituted, which will be summarized in the sub- one dollar Fuzzy computing event handler for the cognition, cognition was immediately effective search, memory, recall and other sub-storage processing, and it is stored in the memory bank model. But at the same storing sub-storage has the potential to trigger computing forgotten child, leading to partial cognition of the body is removed from storage. Bank is a row variable storage array, invalid cell does not occupy storage capacity, thus ensuring limited storage capacity to store as much cognition. The results also proved that brain memory model based on a dollar Fuzzy event to simulate the human brain induction, memory, memories, forgotten and other storage functions. Thus, the brain storage model based on a metsingle fuzzy cognitive unit event is an ideal brain storage model for research in computer science.

\section{ACKNOWLEDGMENTS}

This paper was supported by the Anhui Provincial Natural Science Foundation (1508085MF124).

\section{References}

[1] Harder P., Togeby O. Pragmatics, cognitive science and connectionism, Journal of Pragmatics, 20th. 2016, vol.20, pp.467-492.

[2] Valdas Noreika, Leila Jylhänkangas, Levente Móró. Consciousness lost and found: Subjective experiences in an unresponsive state, Brain and Cognition, 3rd. 2011, vol. 3, pp.327-334.

[3] Lara G Chepenik, Fei Wang, Linda Spencer, et al. Structure - function associations in hippocampus in bipolar disorder, Biological Psychology, 1st. 2012, vol.90, pp.18-22.

[4] Freeman Walter J, Ahlfors Seppo P, Menon Vinod. Combining fMRI with EEG and MEG in order to relate patterns of brain activity to cognition, International journal of psychophysiology,1st. 2009, vol.73, pp.43-52

[5] Petter Marklund, Peter Fransson, Roberto Cabeza, et al. Sustained and Transient Neural Modulations in Prefrontal Cortex Related to Declarative Long-Term Memory, Working Memory, and Attention, Cortex, 1st. 2007, vol.43, pp.22-37.

[6] Kang Feng., Zhaoyin Yuan. Cognitive Computation Based on Single Unit Fuzzy Event, Revista de la Facultad de Ingeniería,11th. 2016, vol. 31,pp.15-24. 